\title{
Ileocolic Intussusception Prolapsing from the Rectum in Von Recklinghausen's Disease
}

\author{
Marlén Alvite Canosa ${ }^{*}$, Leticia Alonso Fernández, Lucía Dorado Castro, Rocío González López, \\ Ana Álvarez Gutiérrez, Inmaculada Monjero Ares, José Conde Vales, Félix Arija Val \\ General Surgery Department of Hospital Universitario Lucus Augusti, Lugo, Spain \\ Email: *marlenqx@hotmail.com
}

Received May 28, 2012; revised July 1, 2012; accepted July 14, 2012

\begin{abstract}
Intussusception is a paediatric condition that rarely presents in adults. We report an exceptional case of ileocolic intussusception prolapsing from the rectum in an adult with Von Recklinghausen's disease. A 31-year-old man with Von Recklinghausen's disease presented to emergency department with a history of severe abdominal pain, vomits and rectal bleeding. Abdominal computed tomography showed intestinal obstruction probably due to a sigmoidorectal intussusception. Ileum, appendix, cecum and ascending colon were found to be intussuscepting through transverse, descending and sigmoid colon and prolapsing from the rectum during an emergent laparotomy. A right hemicolectomy was performed. An anatomical pathology examination revealed a neurofibroma of the appendix as lead point for intussusception. Intussusception in adults requires early surgical resection regardless of the nature of the initial case. Neurofibroma of the appendix is very rare; although it is benign, prompt resection is recommended because of a high risk of appendicitis and malignant transformation.
\end{abstract}

Keywords: Adult Intussusceptions; Neurofibromatosis; Von Recklinghausen’s Disease; Appendiceal Neurofibroma

\section{Introduction}

Intussusception is defined as the telescoping of a segment of the gastrointestinal tract into an adjacent one. Although intestinal intussusception is common in children, it is rare in adults. Adult intussusception represents $1 \%$ of all bowel obstructions and $5 \%$ of all bowel intussusceptions, and approximately $90 \%$ is secondary to a pathological anatomical condition [1].

Although it is generally accepted that adult intussusception requires surgical resection, the extent of resection and the question of whether the intussusception should be reduced remain controversial.

The aim of this report is to present a rare case of ileocolic intussusception prolapsing from the rectum due to appendiceal neurofibroma in an adult patient with Von Recklinghausen's disease.

\section{Clinical Case}

A 31-year-old man with Von Recklinghausen's disease or neurofibromatosis type 1 (NF1) presented to emergency department with a 24-hour history of severe abdominal pain, vomits and rectal bleeding. He had a twomonth history of diarrhoea and colic abdominal pain.

\footnotetext{
${ }^{*}$ Corresponding author.
}

On examination, the patient had characteristic café-aulait spots and subcutaneous neurofibromas, and distended abdomen with generalized pain. On rectal examination, an irregular soft mass non-contiguous with the anus was prolapsed.

Abdominal computed tomography (CT) showed intestinal obstruction probably due to a sigmoidorectal intussusception (Figure 1). An exploratory laparotomy was indicated. Ileum, appendix, cecum and ascending colon were found to be intussuscepting through transverse, descending and sigmoid colon and prolapsing from the rectum (Figure 2). This extensive intussusception was reduced using bimanual pressure, and revealed the presence of an appendiceal tumour acting as lead point for the intussusception (Figure 3). A right hemicolectomy was performed.

Postoperative recovery was uneventful. Histology confirmed a neurofibroma of the appendix as lead point for intussusception.

\section{Discussion}

In contrast to paediatric intussusception, in adults the presentation is often subacute or even chronic. The characteristic paediatric presentation triad of abdominal pain, palpable abdominal mass and bloody stool was only seen in $10 \%$ of cases, so in adults the correct diagnosis is often 

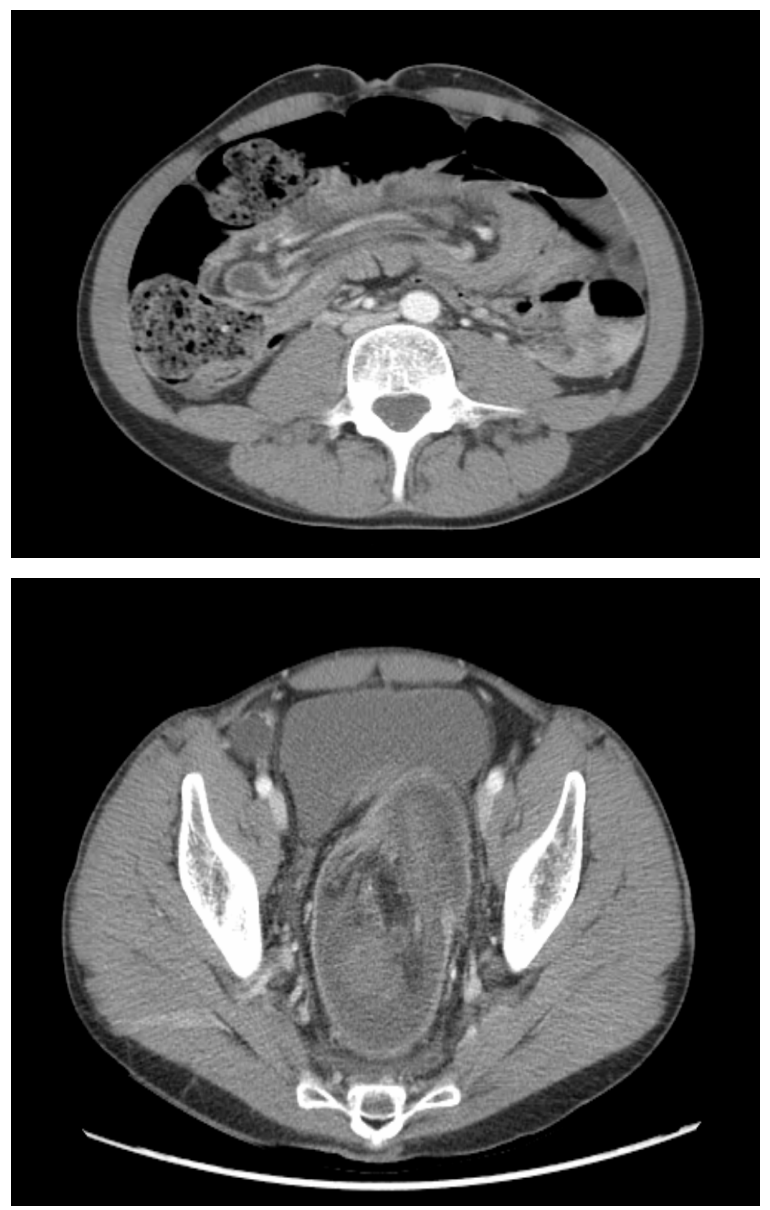

Figure 1. Abdominal computed tomography: intestinal intussusception prolapsing through the rectum.

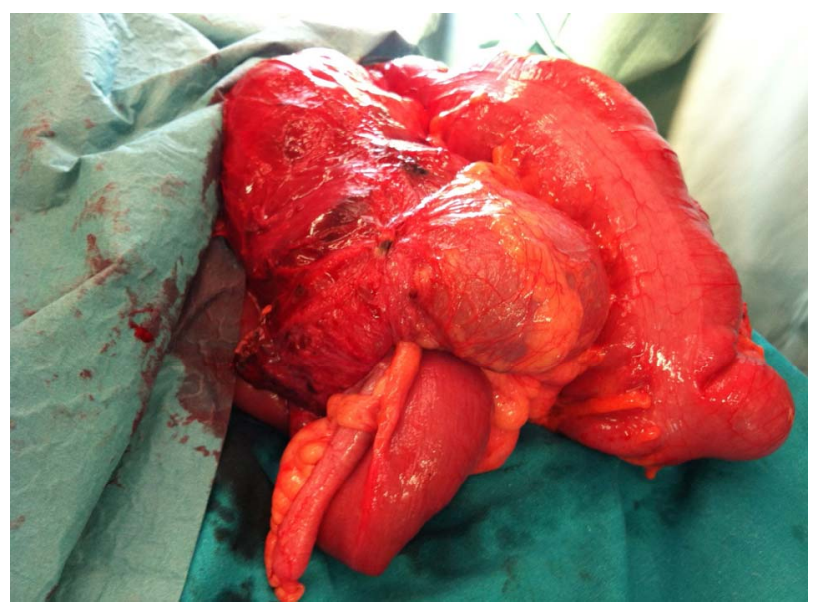

Figure 2. Surgical view: ileum, appendix, cecum as ascending colon implicated in the intussusception after reduction.

unsuspected and it is typically established only during laparotomy. Surgery is mandatory in the treatment of adult intussusception [2]. Adult intussusception often presents with nonspecific symptoms. Preoperative diagnosis remains difficult and the extent of resection, and whether

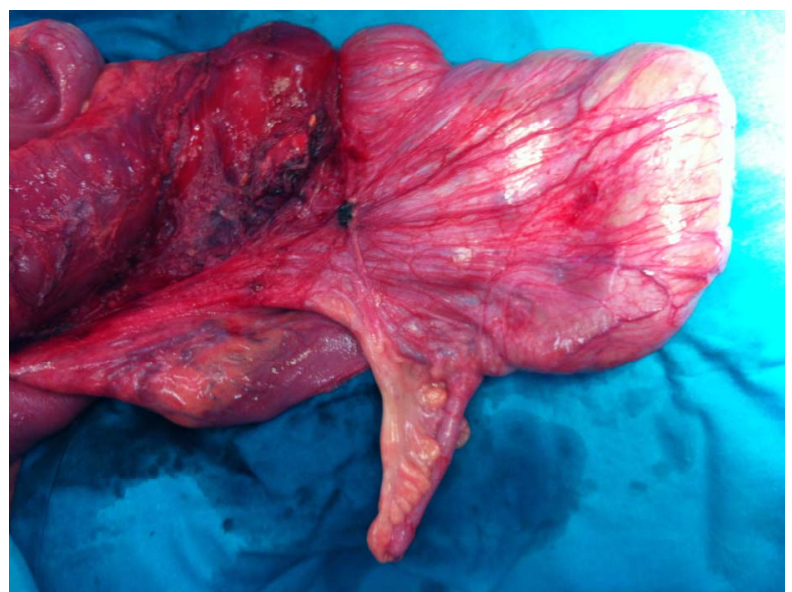

Figure 3. Neurofibroma of the appendix acting as lead point for the intussusception.

the intussusception, should be reduced remains controversial. Some authors suggest that if the underlying etiology and/or the lead point is suspected to be malignant, or if resected area required without reduction is not massive, an en bloc resection of the intussusception should be considered [1-3].

NF1, known as Von Recklinghausen disease, is one of the most common inheritable disorders with an autosomal dominant transmission, an incidence of 1:3000 and an extremely variable clinical expression. Gastrointestinal involvement in NF1 is an uncommon entity, fewer than $25 \%$ of patients with NF1 developed intestinal tumours. Abdominal localizations involve five categories of tumours: neurofibromas, pheochromocytomas, GIST, carcinoids and malignant peripheral nerve sheath tumour [4].

Neurofibromas represent the most common neoplasm occurring within the abdominal cavity and the gastrointestinal tract of NF1. Frequently, they present paraspinal, sacral of mesenterial localization. Neurofibroma of the appendix is very rare, even in patients with NF1; although it is benign, prompt resection is recommended because of a high risk of appendicitis and malignant transformation [5].

\section{Conclusion}

The present case of ileocolic intussusception prolapsing from the rectum in an adult due to neurofibroma of the appendix is exceptional and to our knowledge no case has been described in the literature.

\section{REFERENCES}

[1] N. Wang, X. Y. Cui, Y. Liu, J. Long, Y. Xu, R. Guo and K. Guo, "Adult Intussusception: A Retrospective Review of 41 Cases,” World Journal of Gastroenterology, Vol. 15, No. 26, 2009, pp. 3303-3308. doi:10.3748/wjg.15.3303

[2] B. Hanan, T. R. Diniz, M. M. P. Da Luz, S. A. Da 
Conceicao, R. G. Da Silva and A. Lacerda-Filho, "Intussusception in Adults: A Retrospective Study," Colorectal Disease, Vol. 12, No. 6, 2010, pp. 574-578. doi:10.1111/j.1463-1318.2009.01865.X

[3] R. Chen, H. Zhao, X. Sang, Y. Mao, X. Lu and Y. Yang, "Severe Adult Ileosigmoid Intussusception Prolapsing from the Rectum: A Case Report," Cases Journal, Vol. 1, 2008, p. 198. doi:10.1186/1757-1626-1-198

[4] U. Basile, G. Cavallaro, A. Polistena, S. Giustini, G. Or- lando, D. Cotesta, L. Petramala, C. Letizia, S. Calvieri and G. De Toma, "Gastrointestinal and Retroperitoneal Manifestations of Type 1 Neurofibromatosis," Journal of Gastrointestinal Surgery, Vol. 14, No. 1, 2010, pp. 186194. doi:10.1007/s11605-009-0940-5

[5] S. Sugimoto, M. Takahashi, K. Nakagawa, T. Yabuno, F. Kito and S. Yoshida, "A Case of Neurofibroma of the Appendix in Von Recklinghausen Disease,” Nihon Shokakibyo Gakkai Zasshi, Vol. 108, No. 1, 2011, pp. 68-73. 